\title{
A Comparitive Approach of Spatial Domain over Frequency Domain for Image Superresolution Application
}

\author{
Sanket B. Kasturiwala ${ }^{1}$, Dr. S.A.Ladhake ${ }^{2}$ \\ ${ }^{I}$ (Asst. Prof., Department of Electronics \& Telecommunication Engg.,SIPNA COET,Amravati, INDIA) \\ ${ }_{2}^{2}$ (Principal, Sipna, College of Enggineering \& Technology, Amravati, Maharashtra, INDIA)
}

\begin{abstract}
Super-resolution aims to produce a high-resolution image from a set alone or more low-resolution images by recovering or inventing plausible high-frequency image content. Typical approach is try to reconstruct a high-resolution image using the sub-pixel displacements of several low-resolution images, usually regularized by a generic smoothness prior over the high-resolution image space. Throughout this paper, a higher resolution image is defined as an image with more resolving power.

Super Resolution consists of two main steps: image registration and image reconstruction. Precise alignment of the input images is one of the important terms. In this paper, we have implemented and tested motion estimation algorithms and image reconstruction algorithms in spatial domain as well as in frequency domain in order to study their analytical parameters and a high resolution image is created by using bicubic interpolation over the prealiased images. Also the experimental as well as analytical results of this paper are successful in spatial domain as per the application of image superresolution.
\end{abstract}

Keywords: Superresolution, Image registration, Image Reconstruction.

\section{Introduction}

Cambridge International Advanced Learner's dictionary defines 'Superresolution' as 'the ability of a microscope, or a television or computer screen, to show things clearly and with a lot of detail:'

Superresolution refers to the reconstruction methods that can be applied to obtain an image with higher spatial resolution through the use of several lower-resolution set of (LR) images. Super-Resolution (SR) is a process by which a number of LR images are combined into a single HR image, which has a greater resolving power. SR is not only useful to enhance the resolving power of an image; it can also, to some extent, reduce the aliasing noticeably.

On one hand, increasing the number of pixels on a chip via reducing the pixel size is limited by the existence of shot noise. On the other hand, increasing the chip size is deemed ineffective due to the existence of a large capacitance that slows the charge-transfer rate[ 5]. Super resolution (SR) provides cost effective alternatives for boosting the quality of the image without additional hardware cost.

The idea of superresolution was first introduced in 1984 by Tsai and Huang [1] formulate if same image restoration of band limited signals based on the spatial aliasing effect.

In the literature, super-resolution is treated as an inverse problem, where the high-quality and highresolution image to be obtained is linked to the under sampled images by a series of operators such as warping, blur, decimation and additive noise as per the work presented in projection onto convex sets (POCS) approach, iterative back-projection maximum a posteriori (MAP) estimation, etc. [6].

In almost all above methods, in order for the high-resolution image to be reconstructed, the blur and the motion operators should be known in advance. Although the motion parameters are estimated a priori to some extent, as known to the designer, the blur operator is just assumed to be in hand. But this is mostly impossible in practice. Either the blur parameters must be estimated or the high-resolution image must be constructed without the need for the blur.

Most superresolution methods are composed of two main steps: first all the images are aligned in the same coordinate system in the registration step, and then a high-resolution image is reconstructed from the irregular set of samples. In this second step, the camera point spread function is often taken into account. SuperResolution refers to recovering high resolution data from images that due to mis-focus, compression or other forms of distortion have lost the data that were originally embedded in the higher frequencies of the image, and hence are now given as low resolution images.

The focus of this research paper mainly on based on the two major, and to some extent, independent challenges in superresolution imaging. First, the difference between the low resolution input images needs to be known precisely. This difference can have many origins: camera motion, change of focus, a combination of these two, and so forth. We will consider images that differ by a planar motion. Therefore, the first challenge corresponds to having a precise knowledge of the motion prameters. An error in the motion estimation translates almost directly in to a degradation of the resulting high resolution image. The artifacts caused by an incorrectly 
aligned image are visually much more disturbing than the blurring effect from interpolating only one image. The second challenge is to apply the information obtained from the different registered images to their construction of a sharp high resolution image. Thus, no point spread function (PSF) is taken in to account in the reconstruction.

In this paper, we have implemented image registration algorithm such as Vandewalle et al in frequency domain and Keren et al in spatial domain for motion estimation analysis which gives the rotaion as well as sub pixel shift of low resolution images and image reconstruction algorithm such as interpolation, iterative backpropogation and robust super resolution algorithm.

The superresolution algorithm we have tested in this paper reconstructs an image with almost respective resolution factor in both dimensions from four aliased images. The four low-resolution images are necessarily under sampled.

We compare our approach in a simulation to other spatial domain and frequency domain registration algorithms along with reconstruction algorithms. We find that spatial domain algorithm can better estimate shift and rotation parameters than the other methods, in particular, when some strong directionality is present in the image.

\section{Superresolution Model}

The goal of SuperResolution is to increase the resolution of an image by using many images very similar to it. Note that in this paper, a higher resolution image is not only an image that has more pixels, but is an image that has a greater resolution power (i.e. more details are visible). From these similar pictures of the same scene, where the camera has moved very slightly, we can gather all the information necessary that will in turn enable us to reconstruct a higher resolution image.

Fig. 1 illustrates the image degradation model. In order to treat the most general case, it is assumed that each measurement is the result of different blur, noise, motion, and decimation parameters. Translating the above description to an analytical model, We get

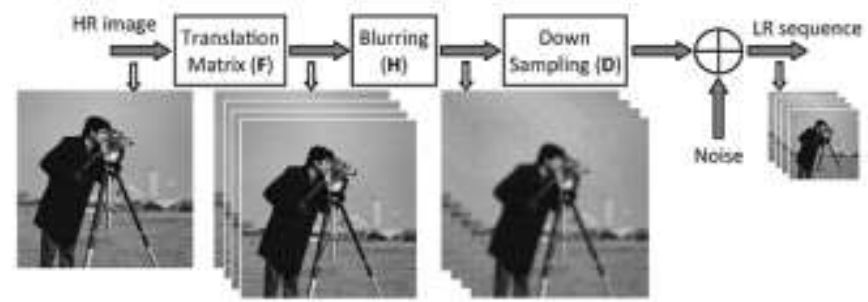

Fig. 1: Degradation Model.

The $n_{k}$ parameter represents the additive gaussian noise. The above restoration problem can be formulated in terms of the following equation:

$$
y_{k}=H \cdot x+n_{k} \text { for } 1 \leq k \leq p .
$$

Where, $H=D B_{k} M_{k}$.

The solution to the above discussed problem can be illustrated form fig. 2.

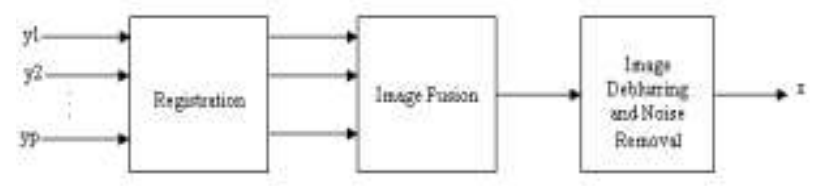

Fig. 2: Image Restoration process for Superresolution.

SuperResolution consists of two main steps: image registration and image reconstruction. The first estimates the motion between the different LR pictures, while the latter uses this information in order to reconstruct the high resolution image by using image fusion and image deblurring technique as shown in fig. 2 .

\section{Image Registration}

A possible application of the proposed image registration algorithm is that of a user holding his digital camera in his hands while manually or automatically taking a series of four shots of a scene within a short period of time. The small vibrations of the user's hands during image capture are sufficient to reconstruct a high resolution image. 
As per the superresolution imaging analysis is concerned, there are two main domain i.e. frequency domain and spatial domain approach for image registration.

In our experiments, we found that the results of the spatial domain algorithm (Keren at al.) are very much better in visual quality as well as in analytical parameter also than the frequency domain algorithm (Vandewalle et al.), which typically failed to adequately register all four images. Super-resolution restoration is critically dependent on accurate, sub-pixel motion estimation.

In the early stages, most of the research work is carried out under frequency domain approach but as more general degradation models were considered; later research has tended to concentrate almost exclusively on spatial domain formulations.

By the nature of frequency domain, fourier transform methods are limited to only global motion models.

\subsection{Motion Estimation}

Motion estimation techniques should only return the translation and rotation information, in the following format:

[Delta est, phi est $]=$ method $(\ldots)$

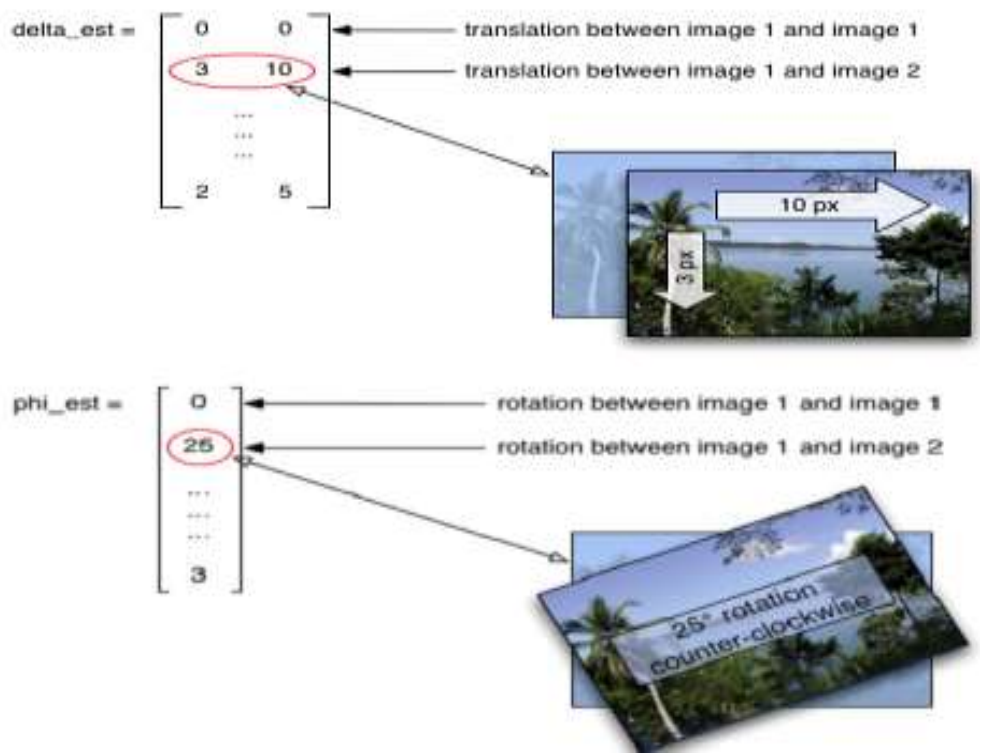

Fig.3: Fundamental Motion Estimation Model

Intuitively, each LR-observed image represents a subsampled (i.e. aliased) version of the original scene.Due to the subpixel shifts, however, each observed image contains complementary information. With exact knowledge of the shifts, the observed image scan be combined to remove the aliasing and generate a higher resolution image. If we assume that the resolution of this HR image is such that the Nyquist sampling criterion is satisfied, this HR image then represents an accurate representation of the original (continuous) scene.

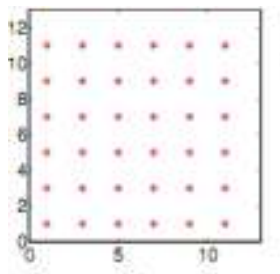

(a)

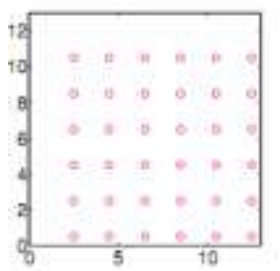

(d)

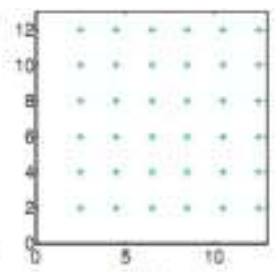

(b)

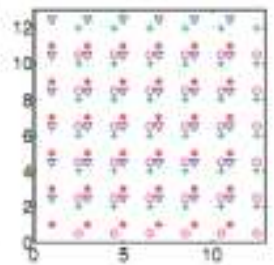

(c)

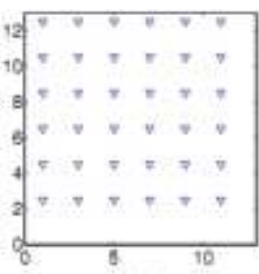

(c)

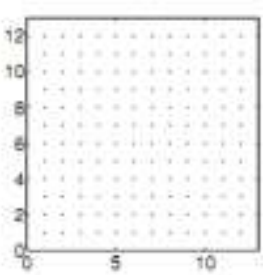

(f)

Fig. 4: (a)-(d) Relationship between LR images; (e) irregularly sampled HR image; (f) the regularly sampled HR image. 
Fig.4 (a)-(d) represents the relationship between the four undersampled LR images and (e) represents the irregularly sampled HR image from four LR while (f) represent the uniformly sampled HR image.

\subsubsection{Frequency Domain}

Most of the frequency domain registration methods are based on the fact of two shifted images differ in frequency domain by a phase shift only, which can be found from phase correlation methods. Basically, we have tested a frequency domain vandewalle et al. algorithm for planar motion estimation in which a shift in the space domain is translated into a linear shift in the phase of the image's Fourier Transform. Similarly, a rotation in the space domain is visible in the amplitude of the Fourier Transform. Hence, the Vandewalle et al. motion estimation algorithm computes the images' Fourier Transforms and determines the 1-D shifts in both their amplitudes and phases. One advantage of this method is that it discards high-frequency components, where aliasing may have occurred, in order to be more robust. Especially planar motion parallel to the image plane is allowed. The motion can be described as a function of three parameters: horizontal and vertical shifts, $\Delta x 1$ and $\Delta x 2$, and a planar rotation angle $\theta$ separately.

Assume we have a reference signal $f_{1}(x)$ and its shifted and rotated version $f_{2}(x)$ as shown in (2):

$$
f_{2}(x)=f_{1}(R(x+\Delta x))
$$

Where,

$$
x=\left[\begin{array}{l}
x_{1} \\
x_{2}
\end{array}\right], \Delta x=\left[\begin{array}{l}
\Delta x_{1} \\
\Delta x_{2}
\end{array}\right], R=\left[\begin{array}{cc}
\cos \theta & -\sin \theta \\
\sin \theta & \cos \theta
\end{array}\right]
$$

This can be expressed in fourier domain as

$$
\begin{aligned}
& F_{2}(u)=\iint_{x} f_{2}(x) e^{-j 2 \Pi u^{T} x} d x \\
& =\iint_{x} f_{1}(R(x+\Delta x)) e^{-j 2 \Pi u^{T} x} d x \\
& =e^{j 2 \Pi u^{T} \Delta x} \iint f_{1}\left(R x^{\prime}\right) e^{-j 2 \Pi u^{T} x^{\prime}} d x^{\prime}
\end{aligned}
$$

Where, $F_{2}(u)$ is fourier transform of $f_{2}(x)$ and the coordinate transformation $x^{\prime}=x+\Delta x$ as shown in (4). After another transformation we get,

$$
\left|F_{2}(u)\right|=\left|F_{1}(R(u))\right|
$$

Where, $\left|F_{2}(u)\right|$ represent a rotated version of $\left|F_{1}(u)\right|$ over the same angle $\theta$. Thus, $\left|F_{2}(u)\right|$ and $\left|F_{1}(u)\right|$ mostly depends on the rotation angle $\theta$. So, in this algorithm first of all rotation angle is estimated.

The rotation angle between $\left|F_{I}(u)\right|$ and $\left|F_{2}(u)\right|$ can be computed as the angle $\theta$ for which the Fourier transform of the reference image $\left|F_{l}(u)\right|$ and the rotated Fourier transform of the image to be registered $\left|F_{2}\left(R_{\theta} u\right)\right|$ have a maximum correlation. But for mainly the low frequencies which generally contain most of the energy introduces large approximation errors. And mostly fails to obtain rotation angle accurately for most of the images as shown in table.

A shift estimation parameter $\Delta x$ can be computed as the linear phase difference between $F_{1}(u)$ and $F_{2}$ (u). One advantage of this algorithm is that the high frequency noise component is removed along with aliasing effect.

As per the experiments, result from this frequency domain algorithm is worse than that of spatial domain Keren et al. algorithm. This algorithm is applicable if and only if some directionality is present in the image.

\subsubsection{Spatial Domain}

Spatial domain methods generally allow for more general motion models, such as homographies. They can be based on the whole image or on a set of selected corresponding feature vectors, as discussed es in their RANSAC algorithm [15]. In this paper, we have tested a Keren ET tal. Spatial domain algorithm for motion estimation estimation which shows a very good observation as well as visual results as shown in table. Keren et 
al is an iterative planar motion estimation algorithm based on Taylor expansions. A pyramidal scheme is used to increase the precision for large motion parameters.

To obtain an estimate of HR image, from the spatio temporal relationship of the HR and LR sequences need to be modeled and cast within the Bayesian framework. This mathematical Bayesian approximation is a very much accurate technique due to which in general spatial domain is preferred for hyper spectral image restoration applications.

Thus, in this modeling of SR technique HR images $f_{k}$ and motion vector $\mathrm{d}$ are treated as a samples of random fields, with corresponding prior distribution that models our knowledge about the nature of the original HR image and motion vector.

The observation o which is a function of $f_{k}$ and $d$ is also treated as a sample of random field with corresponding conditional distribution that models the algorithm to process to obtain o from $f_{k}$ and $\mathrm{d}$.

The joint distribution modeling the relationship between the observed data and the unknown quantities of the SR problem becomes

$$
P\left(f_{k}, d \mid o\right)=P(f k, d) P(o \mid f k, d)
$$

$\boldsymbol{P}(\boldsymbol{o} \mid f k, d)$ Represents the likelihood of the observation from (5). The Bayesian inference modeling is performed using the posterior

$$
P\left(f_{k}, d \mid o\right)=\frac{P\left(f_{k}, d\right) \cdot P\left(o \mid f_{k}, d\right)}{P(o)}
$$

In its simplest form, Bayesian inference provides the maximum a posteriori (MAP) solution represented $\hat{f}_{k}$ and $\hat{d}$, that maximize the posterior probability distribution,

$$
\begin{aligned}
\hat{f_{k}} \cdot \hat{d} & =\arg \max _{f_{k}, d} P\left(f_{k}, d \mid o\right) \\
& =\arg \max _{f_{k}, d}\left\{\frac{P\left(f_{k}, d\right) P\left(o \mid f_{k}\right)}{P(o)}\right\} . \\
& =\arg \max _{f_{k}, d}\left\{P\left(f_{k}, d\right) P\left(o \mid f_{k}, d\right)\right\} . \\
& =\arg \max _{f_{k}, d}\left\{-\log P\left(f_{k}, d\right)-\log P\left(o \mid f_{k}, d\right)\right\} .
\end{aligned}
$$

Depending on the prior and condition probability density functions ( $p d f$ ), it may be difficult to find out analytical solution for (7) and (9), so numerical solutions are needed.

According to the MAP estimator, the additive noise, the measurements, and the ideal image are all assumed stochastic signals. Bayesian approach provides a flexible and convenient way to model a priori knowledge concerning solution. Even with the numerical solutions, the major problem in the optimization is the simultaneous estimation of the variables $f_{k}$ and $d$.

The HR image $f_{k}$ is then estimated by assuming that displacement estimates are exact.

$$
\hat{f}_{k}^{q+1}=\arg \max _{f_{k}} P\left(f_{k}, \hat{d}^{q}\right) P\left(o \mid f_{k}, \hat{d}^{q}\right)
$$

From (10), the iteration index $q$ is then set to $q+1$ and the solution in eq. are found again and again till the convergence occurs. In order to obtain an estimate of HR image the error $e_{l, k} \quad l=1,2,3, \ldots \ldots \ldots ., \mathrm{L}$ in the formation model below is minimized.

$$
g_{l}=A_{l} . H_{l} . C\left(d_{l, k}\right) f_{k}+e_{l, k} \quad l=1,2,3, \ldots \ldots, \mathrm{L} .
$$

The downsampling factor $A_{l}$, the blur matrix $H_{l}$ and $C\left(d_{l, k}\right)$ the motion compensation matrix that rotate the observed LR image $g_{l}$ to the HR image $f_{k}$ as shown in (11).

Most of the HR motion estimation methods are used for uncompressed LR observation $\left(\mathrm{g}_{1}, \mathrm{~g}_{2}, \mathrm{~g}_{3}, \ldots \ldots, \mathrm{g}_{\mathrm{L}}\right)$, first, interpolating to obtain $\mathrm{u}$, an estimate of the original HR sequence $\mathrm{f}$ and then find the displacement vector $d_{l, k(x, y)}$ satisfying (12)

$$
u_{l}(x, y)=u_{k}\left(x+d_{l, k}^{x}(x, y), \quad y+d_{l, k}^{y}(x, y)\right)
$$

There are different methods to reconstruct $\mathrm{u}$. Most HR motion estimation methods to estimate $d_{l, k}$ from the unsampled sequence $u$, particularly those derived in frequency (fourier) domain, are based on the assumption of purely translational image motion.

This translation motion model corresponds to

$$
d_{l, k}^{x}(x, y)=p 1 \quad \text { And } \quad d_{l, k}^{y}(x, y)=p 2 .
$$

either for all pixels in the $l^{\text {th }}$ image or for pixels within a given block or region in that image. Keren et al method is used for estimating translation motion model. The next level of complexity in modeling the image motion is 
to assume that images $u_{l}$ and $u_{k}$ (or regions within them) as shown in (13) are related by a horizontal shift ' $a$ ', a vertical shift ' $b$ ' and a rotation angle ' $\theta$ ' around the origin

$$
u_{l}(x, y)=u_{k}(x \cos \theta-y \sin \theta+a \quad y \cos \theta+x \sin \theta+b)
$$

The estimation of the rotation angle and the translation is modeled using an affine transformation (of which translation, rotation and scaling are particular cases).

$$
u_{l}(x, y)=u_{k}(a x+b y+c, \quad d x+e y+f)
$$

Where, a, $b, c, d, e$ and $f$ are the parameters to be estimated from (14). This model has been used in SR problems, for instance, in bilinear transformation.

$$
u_{l}(x, y)=u_{k}(a x+b y+c x y+d, \quad e x+f y+g x y+h)
$$

Where, $a, b, c, d, e, f, g$ and $h$ are the parameters to be estimated from (15).

Then the affine transformation from (15) using warp

$$
\begin{aligned}
& W(x ; p)=\left[\begin{array}{l}
x+(a-1) x+b y+c \\
y+d x+(e-1) y+f
\end{array}\right] \\
& \text { With } p=\left[\begin{array}{llllll}
a-1 & b & c & d & e-1 & f
\end{array}\right]_{\text {from (18). }}^{t}
\end{aligned}
$$

The vector $\mathrm{p}$ can be region dependant or pixel dependant. We denote by $B$ the image region where $p$ is assumed to be a constant.

However, this algorithm, do not requires any directionalities should present in the images. A robust search procedure such as the random sample consensus (RANSAC) spatial domain algorithm which is used for the estimation of the parameters of a geometric transformation by automatic detection and image features with subpixel accuracy which returns a highly accurate estimate of the transformation. When geometric transformation is planar homography which is given as a plane projective transformation. But this algorithm is not useful for video sequence estimation.

\section{Image Reconstruction}

To reconstruct the high-resolution image, we apply bicubic interpolation on a high-resolution grid. The super-resolution algorithm we propose reconstructs an image with almost double resolution in both dimensions from four aliased images. The four low-resolution images are necessarily under sampled.

In this paper, we have tested two algorithms for image reconstruction i.e. iterative backprojection and robust superresolution.

The idea behind Iterated Back Projection [6] is to start with a rough estimation of the HR image, and iteratively add to it a "gradient" image, which is nothing else than the sum of the errors between each LR image and the estimated HR image that went through the appropriate transforms (given by the motion estimates).

Robust Super Resolution [12] is a more robust version of the above Iterated Back Projection. The only difference resides in the computation of the gradient, which is not given by the sum of all errors, but by the median of all errors. This brings robustness against outliners in the LR images. Zomet et al.[11] improved the results obtained with typical iterative back projection algorithms by taking the median of the errors in the different backprojected images. This proved to be more robust in the presence of outliers. Farsiu et al. [12] proposed a new and robust super-resolution algorithm. An optical low pass filter is applied in order to reduce high frequency noise component, blur and aliasing effect during image restoration process.

The complete image registration and image reconstruction process is carried out by multiplying tuky window which is one of the digital filter reduces the noise component from the images.

\section{Experimental Analysis}

The camera was held manually in approximately the same position while taking the pictures, which caused small shifts and rotations between the images. Images are captures by using low resolution mobile cameras. Aliasing is present in the high frequency regions of the images. The different registration algorithms in frequency domain such as Vandewalle algorithm and in spatial domain such as Keren algorithm are then applied to these images and a high resolution image is reconstructed by using iterative backpropogation (IBP) algorithm and using robust superresolution (RSR) algorithm.

Because the original images are already relatively large, the required additional upsampling and interpolation require too much memory to be performed on a regular computer. The upsampling is therefore is limited in this case and the shifts are computed upto pixel level. Because the exact motion parameters are 
unknown, it is only possible to compare visually the different reconstructed images. Most aliasing has been removed in both reconstructed images.

Testing and implementation of the image registration and image reconstruction algorithms for superresolution applications are performed under MATLAB environment using various commands from Image Processing Toolbox.

Fig. 6 shows the original low resolution Alternera (a soybean leaf disease) frames with [64x64] resolution. Original frame is interpolated by factor of 4 .

In this case we have applied frequency domain i.e. Vandewalle motion estimation algorithm. Fig. 6 (a) shows the result for iterative backpropogation (IBP) algorithm and Fig. 6 (b) shows the for robust superresolution (RSR) algorithm. Secondly, we have applied spatial domain i.e. Keren motion estimation algorithm. Fig. 6 (c) shows the result for iterative backpropogation (IBP) algorithm and Fig. 6 (d) shows the result for robust superresolution (RSR) algorithm. The final reconstructed image resolution is [256x256].

Fig. 7 shows the original low resolution Soybean Looper (a soybean leaf disease) frames with [64x64] resolution. Original frame is interpolated by factor of 4 .

In this case we have applied frequency domain i.e. Vandewalle motion estimation algorithm. Fig. 7 (a) shows the result for iterative backpropogation (IBP) algorithm and Fig. 7 (b) shows the for robust superresolution (RSR) algorithm. Secondly, we have applied spatial domain i.e. Keren motion estimation algorithm. Fig. 7 (c) shows the result for iterative backpropogation (IBP) algorithm and Fig. 7 (d) shows the result for robust superresolution (RSR) algorithm. The final reconstructed image resolution is [256x256].

Fig. 8 shows the gray scale Lenna image with low resolution frames with [128x128] resolution. Original frame is interpolated by factor of 3 .

Initially, we have applied frequency domain i.e. Vandewalle motion estimation algorithm. Fig. 8 (a) shows the result for iterative backpropogation (IBP) algorithm and Fig. 8 (b) shows the for robust superresolution (RSR) algorithm. At the same time same image frames we have applied for spatial domain i.e. Keren motion estimation algorithm. Fig. 8 (c) shows the result for iterative backpropogation (IBP) algorithm and Fig. 8 (d) shows the result for robust superresolution (RSR) algorithm. The final reconstructed image resolution is [384x384].

Fig. 9 shows the original low resolution Ear Warm (a soybean leaf disease) frames with [64x64] resolution. Original frame is interpolated by factor of 2 .

In this case, also, we have applied frequency domain i.e. Vandewalle motion estimation algorithm. Fig. 9 (a) shows the result for iterative backpropogation (IBP) algorithm and Fig. 9 (b) shows the for robust superresolution (RSR) algorithm. Secondly, we have applied spatial domain i.e. Keren motion estimation algorithm. Fig. 9 (c) shows the result for iterative backpropogation (IBP) algorithm and Fig. 9 (d) shows the result for robust superresolution (RSR) algorithm. The final reconstructed image resolution is [128x128].

From all the above experimental results, we can conclude that though the resolution of the original low resolution fames are increased in frequency domain but visual results gets detiorated as shown in Fig. 5(a), Fig. 7(a) and Fig. 8(a) as compared with image reconstruction using spatial domain approach.

Again, the image reconstruction technique is concerned, visual observation results using robust Superresolution (RSR) Reconstruction are very much better than Iterative Backpropogation (IBP) reconstruction techniques in their respective frequency domain (Vandewalle estimation) and spatial domain (Keren estimation) approach.

\section{Experimental Result}

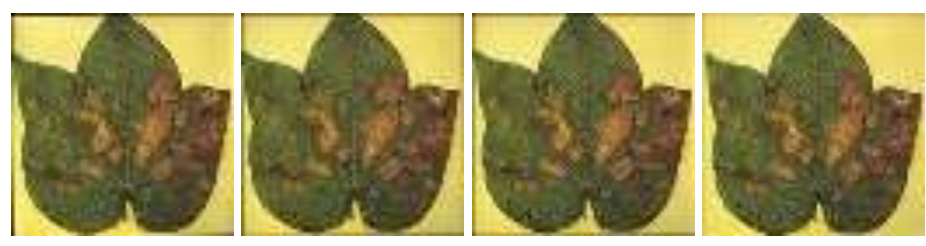

Fig.6: Alterneria: original LR frames [64x64]

Interpolating Factor $=4$ 


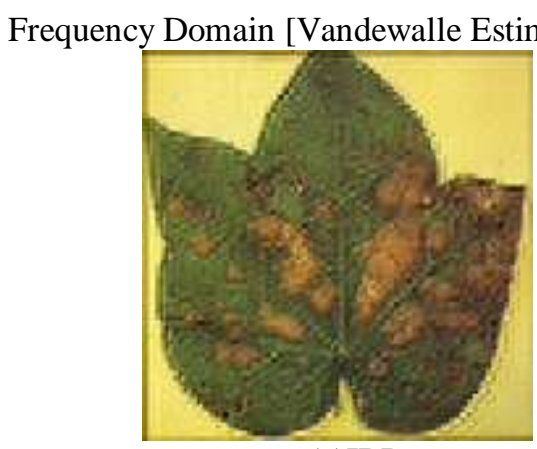

(a)IBP

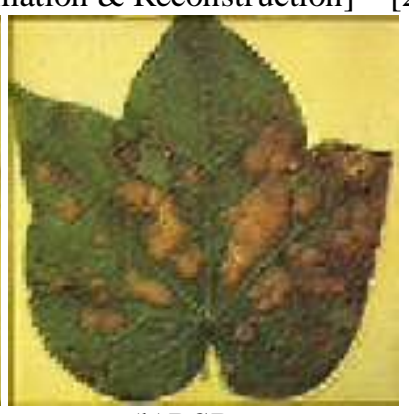

(b)RSR

Spatial Domain [Keren Estimation \& Reconstruction] [256x256]

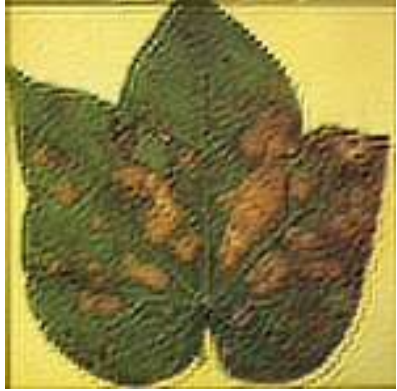

(c) IBP

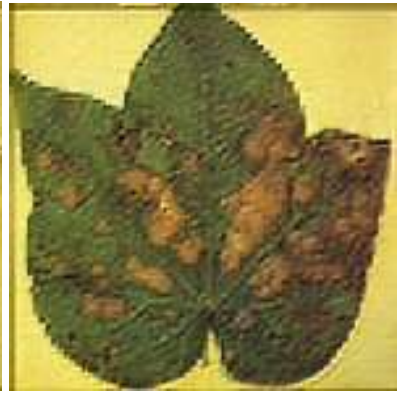

(d)RSR
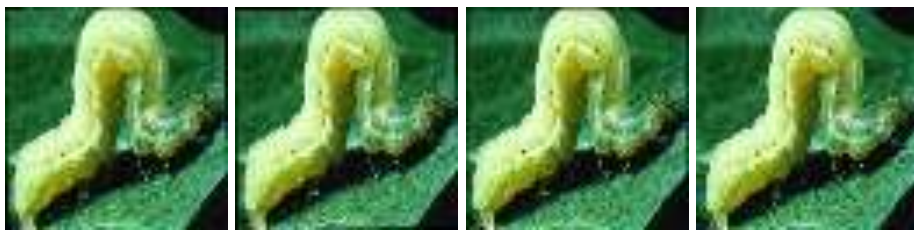

Fig. 7 : Soybean Looper Original Frame Size [64x64] Interpolating Factor $=4$

Frequency Domain [Vandewalle Estimation \& Reconstruction] [256x256]

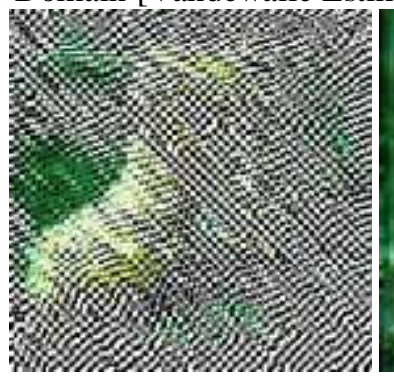

(a)IBP

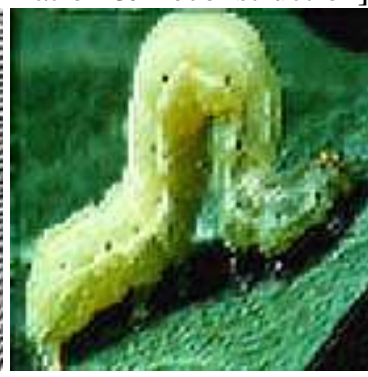

(b)RSR

Spatial Domain [Keren Estimation \& Reconstruction] [256x256]

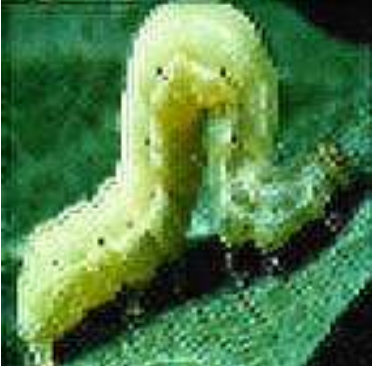

(a)IBP

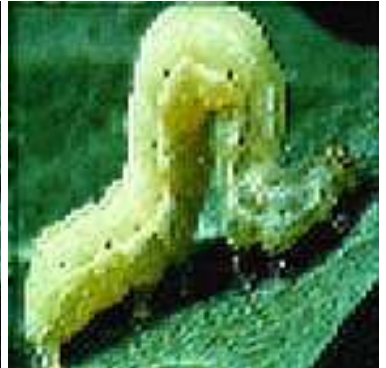

(b)RSR 

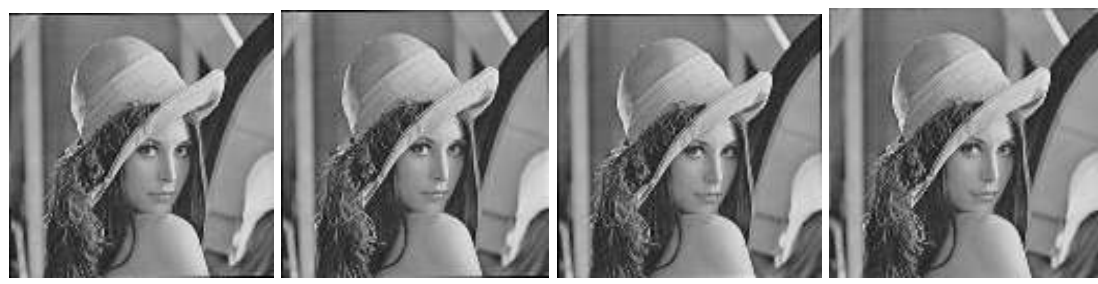

Fig. 8: Lena image. Original Frame size [128x128] Interpolating Factor $=3$

Frequency Domain [Vandewalle Estimation \& Reconstruction]

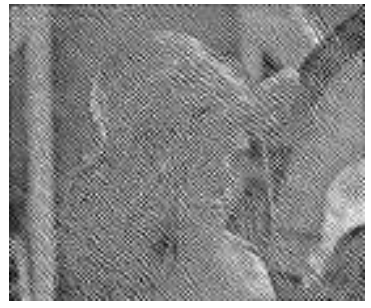

(c)IBP

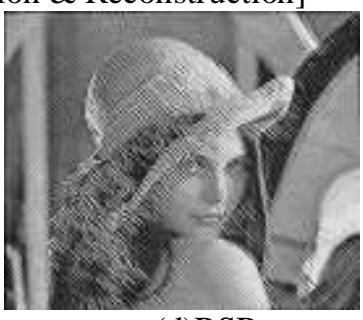

(d)RSR

[384x384]

patial Domain [Keren Estimation \& Reconstruction]

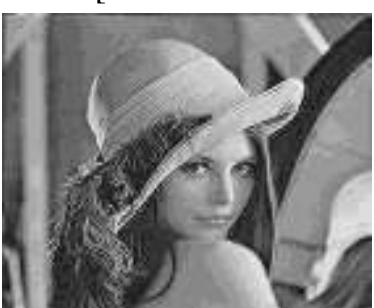

(a)IBP

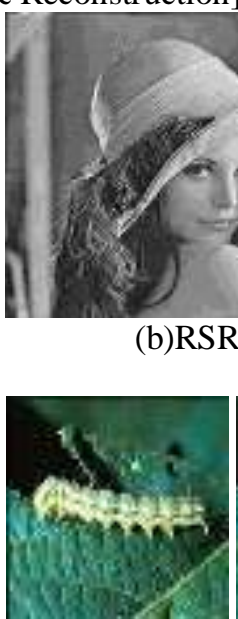

(b)RSR
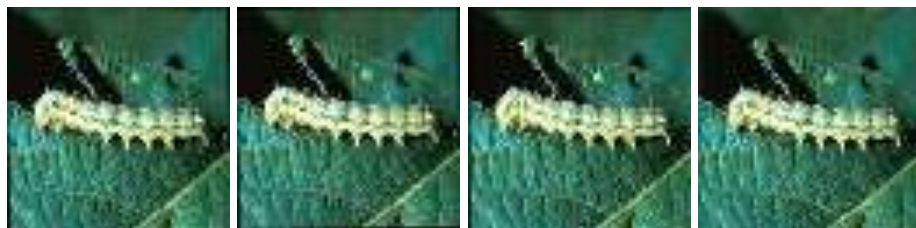

Fig. 9: Ear Warm. Original LR frame [64 x64] Interpolating Factor 2

Frequency Domain [Vandewalle Estimation \& Reconstruction [128x128]

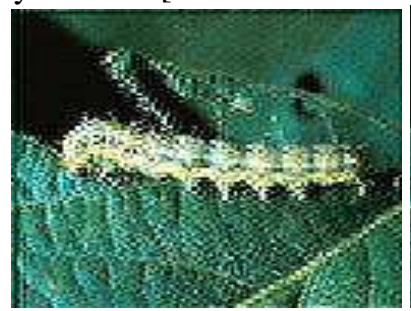

(a)IBP

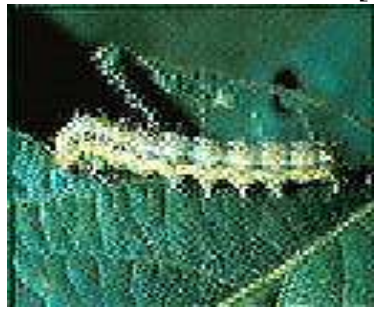

(b)RSR [384x384]

Spatial Domain [Keren Estimation \& Reconstruction [128x128]

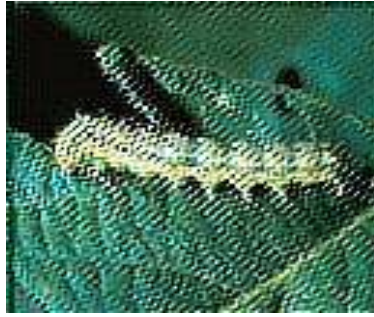

(a)IBP

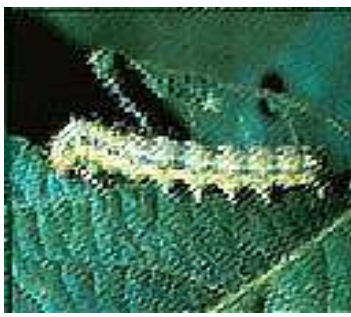

(b)RSR 
Table 2: Frequency Domain (Vandewalle Estimation)

\begin{tabular}{|l|c|c|c|}
\hline \multirow{2}{*}{ LR Images (x4) } & $\begin{array}{c}\text { Avg. } \\
\text { Rotation }\end{array}$ & \multicolumn{2}{|c|}{$\begin{array}{c}\text { Avg. } \\
\text { subpixel Shift }\end{array}$} \\
\cline { 2 - 4 } & $\boldsymbol{\mu}_{\boldsymbol{\theta}}$ & $\boldsymbol{\mu}_{\mathbf{x}}$ & $\boldsymbol{\mu}_{\mathbf{v}}$ \\
\hline Alterneria & 0.06344 & 0.16957 & 0.30668 \\
\hline Ear Warm & 0.2170 & 0.3465 & 0.54033 \\
\hline Soyabean Looper & 0.30154 & 0.3292 & 0.7372 \\
\hline Lena & 0.38679 & 0.33558 & 0.36678 \\
\hline
\end{tabular}

Table 3: Spatial Domain (Keren Estimation)

\begin{tabular}{|c|c|c|c|}
\hline \multirow{2}{*}{ LR Images (x4) } & $\begin{array}{c}\text { Avg. } \\
\text { Rotation }\end{array}$ & \multicolumn{2}{|c|}{$\begin{array}{c}\text { Avg. } \\
\text { subpixel Shift }\end{array}$} \\
\cline { 2 - 4 } & $\boldsymbol{\mu}_{\boldsymbol{\theta}}$ & $\boldsymbol{\mu}_{\mathbf{x}}$ & $\boldsymbol{\mu}_{\mathbf{y}}$ \\
\hline Alterneria & -0.0666 & 0.53926 & 0.26139 \\
\hline Ear Warm & 0 & 0.40238 & 0.6310 \\
\hline Soyabean Looper & -0.03333 & 0.4015 & 0.808021 \\
\hline Lena & 0 & 0.55464 & 0.41487 \\
\hline
\end{tabular}

Table 4: Frequency Domain Vandewalle Image Reconstruction using Iterative Backpropogation (IBP) and Robust SuperResoluton (RSR) algorithm with interpolating factor.

\begin{tabular}{|c|c|c|c|c|c|c|}
\hline \multirow{2}{*}{ LR Images (x4) } & \multirow{2}{*}{$\begin{array}{l}\text { Facto } \\
\mathbf{r}\end{array}$} & \multicolumn{2}{|c|}{ IBP } & \multicolumn{2}{|c|}{ RSR } & \multirow{2}{*}{$\begin{array}{l}\text { Enhanced } \\
\text { Resolution }\end{array}$} \\
\hline & & Iterations & Errors & Iterations & Errors & \\
\hline Alterneria & 4 & 11 & 0.0565 & 14 & 0.02063 & {$[256 \times 256]$} \\
\hline Ear Warm & 2 & 40 & 0.0671 & 33 & 0.06159 & {$[128 \times 128]$} \\
\hline Soyabean Looper(64x64) & 4 & 15 & 0.0201 & 15 & 0.07636 & {$[256 \times 256]$} \\
\hline$(128 \times 128)$ & 3 & 57 & 0.0265 & 50 & 0.0014 & {$[384 \times 384]$} \\
\hline
\end{tabular}

Table 5: Spatial Domain Keren Image Reconstruction using Iterative Backpropogation (IBP) and Robust SuperResoluton (RSR) algorithm with interpolating factor.

\begin{tabular}{|c|c|c|c|c|c|c|}
\hline \multirow{2}{*}{ LR Images (x4) } & \multirow{2}{*}{ Factor } & \multicolumn{2}{|c|}{ IBP } & \multicolumn{2}{c|}{ RSR } & $\begin{array}{c}\text { Enhance } \\
\text { Resolution }\end{array}$ \\
\cline { 3 - 7 } & & Iterations & Errors & Iterations & Errors & 13 \\
\hline Alterneria (64 x 64) & 4 & 0.0392 & 18 & 0.00824 & {$[256 \times 256]$} \\
\hline Ear Warm (64 x 64) & 2 & 16 & 0.0617 & 26 & 0.06452 & {$[128 \times 128]$} \\
\hline Soyabean Looper (64 x64) & 4 & 100 & 0.0226 & 10 & 0.0619 & {$[256 \times 256]$} \\
\hline Lena (128 x 128) & 3 & 10 & 0.0638 & 15 & 0.0410 & {$[384 \times 384]$} \\
\hline
\end{tabular}

Table II shows the tabulated result of average rotation and average subpixel shift estimation for frequency domain approach using Vandewalle estimation algorithm and Table III shows the tabulated result of average rotation and average subpixel shift estimation for spatial domain approach using Keren estimation algorithm. Frequency domain approach using Vandewalle estimation algorithm mostly fails to represent the exact and proper estimation of average rotation of low resolution frames of the input images as compared with spatial domain approach using Keren estimation algorithm. But on the other side, average shift (horizontal and vertical) estimation shows nearly the same result for frequency domain as well as spatial domain estimation technique.

Table IV and Table $\mathrm{V}$ shows the tabulated result for image reconstruction using Iterative Backpropogation (IBP) and Robust Superresolution (RSR) techniques in frequency domain and spatial domain, respectively. From both the table, we observe that the number of iterations required for image reconstruction process using iterative backpropogation (IBP) and robust superresolution (RSR) techniques mostly depends on interpolating factor either for frequency domain or for spatial domain approach. From this we get the idea about interpolating factor that if the interpolating factor is high, it requires somewhat more iterations and much more time for processing.

Table IV and Table $\mathrm{V}$ also shows the very good error rate estimation for iterative backpropogation (IBP) as well as robust superresolution (RSR) image reconstruction techniques for frequency domain (Vandewalle estimation) and for spatial domain (Keren estimation) algorithm. But here for some image superresolution process, the number of iterations and error rate are much smaller either for frequency domain (Vandewalle et al.) or for spatial domain (Keren et al.). The last column of both the tables indicates the 
enhanced resolution of the LR frames with respect to their interpolating factor.Both the reconstruction domains are applicable either for gray level images or for color images.

\section{Conclusion And Future Scope}

Through this research paper, we have reconstructed a superresolution process from a set of LR images captured by low cost low resolution mobile or digital camera and recovered a plausible high frequency image contents from it. Spatial domain analysis using Keren estimation shows very good observational as well as well analytical result. The reconstructed image through spatial domain having more resolving power. Due to this high resolving imaging power, aliasing ambiguity gets eliminated from LR subpixel displacement frames. As that of frequency domain, here in spatial domain there is no requirement of directionality present in the LR frames. Here we can reconstruct superresolution of either gray level or color images also.

In future direction, for high resolution image feature extraction and classification using classifier under spatial domain superresolution tool is the prime aspect. Therefore, this spatial domain superresolution is applicable for agricultural images, hyper spectral satellite images, biomedical images, biometric images also.

\section{Journal Papers:}

\section{References}

[1] R.Y. Tsai and T.S. Huang, "Multiframe image restoration and registration," in Advances in Computer Vision and Image Processing, vol.1, chapter7, pp.317-339, JAI Press, Greenwich, USA, 1984.

[2] M.V.Joshi, S.Chaudhuri and R.Panuganti, "Super-Resolution imaging: use of zoom as a cue," Image and Vision Computing, vol.22, no.14, pp.1185-1196, 2004.

[3] Julien Mairal, Michael Elad, and Guillermo Sapiro, Senior Member, IEEE , "Sparse Representation for Color Image Restoration", IEEE Trans. on Image Proces. vol. 17, no. 1, Jan., 2008.

[4] F.Sroubek, G.Cristobal and J. Flusser, "Simultaneous super-resolution and blind deconvolution", 4th AIP International Conference and the 1st Congress of the IPIA IOP Publishing Journal of Physics: Conference Series 124 (2008) 012048.

[5] S. C. Park, M. K. Park, and M. G. Kang, "Super-resolution image reconstruction - a technical overview", IEEE Signal Process. Magazine, vol. 20, pp. 21-36, May 2003

[6] R. C. Hardie, K. J. Barnard, J. G. Bognar, E. E. Armstrong, and E. A. Watson, "High-resolution image reconstruction from a sequence of rotated and translated frames and its application to an infrared imaging system", Optical Engineering, 37(1), 247-260 (1998).

[7] Merino, M.T. and Núñez, J., "Super-resolution of remotely sensed images with variable-pixel linear reconstruction", IEEE Trans. Geosci. and Remote Sensing, vol.45, pp.1446-1457 (2007).

[8] M. Elad and A. Feuer, "Super-resolution reconstruction of image sequences", IEEE Trans. on Pattern Analysis and Machine Intelligence, 21(9):817 1999.

[9] S.Borman and R.L. Stevenson, "Spatial resolution enhancement of low-resolution image sequences a comprehensive review with directions for future research," Tech. Rep., Laboratory for Image and Signal Analysis (LISA), University of Notre Dame, USA, 1998.

[10] S.Farsiu, M.Robinson, M.Elad, and P.Milanfar, "Fast and robust multiframe superresolution," IEEE Trans. Image Process., vol.13, no.10, pp.1327-1344, Oct.2004.

[11] A.Sanchez Beato and G.Pajares, "Noniterative interpolation based superresolution minimizing aliasing in the reconstructed image," IEEE Trans. Image Process., vol.17, no.10, pp.1817-1826, Oct.2008.

[12] L.Baboulaz and P.L.Dragotti, "Exact feature extraction using finite rate of innovation principles with an application to image superresolution,” IEEE Trans. Image Process., vol.18, no.2, pp.281-298, Feb.2009.

[13] H.Shen, L.Zhang, B.Huang, and P.Li, “A map approach for joint motion estimation, segmentation, and super-resolution,” IEEE Trans. Image Process., vol.16, no.2, pp.479-490, Feb.2007,

[14] M.Chen,"Dynamic content adaptive superresolution," in Proc. Int. Conf. Image Anal. Recog., 2004, vol.3212, pp.220-227.

[15] Y.He, K.-H.Yap, L.Chen and L.P.Chau, "A nonlinear least square technique for simultaneous image registration and superresolution," IEEE Trans. Image Process., vol.16,no.11,pp.2830-2841,Nov.2007.

[16] Fei Zhou,Wen ming Yang, and Qing min Liao "A Coarse-to-Fine Subpixel Registration Method to Recover Local Perspective Deformation in the Application of Image Super-Resolution", IEEE Trans., on Image process, VOL.21, NO.1, Jan.2012.

[17] Z.Ivanovski, L.Karam and G.Abousleman, "Selective Bayesian estimation for efficient superresolution," in Proc.4 ${ }^{\text {th }}$ IEEE Int.Symp. Signal Process. Inf. Technol., Dec.2004,

[18] R.Hardie, "A fast image superesolution algorithm using an adaptive wiener filter," IEEE Trans.Image Process., vol.16,no.12,pp.2953-2964,Dec.2007.

Books:

[19] Rafael C. Gonzalez, Richard E. Woods, Steven L.Eddins, Book- Digital Image Processing using MATLAB.

[20] Sonka,Hlavac and Boyle, Book- Digital Image Processing and Computer Vision.

Proceedings Papers:

[21] D.Keren, S.Peleg, and R.Brada, "Image sequence enhancement using sub-pixel displacements," in Proceedings of IEEE Computer Society Conference on Computer Vision and Pattern Recognition (CVPR'88), pp.742-746,USA,June1988.

[22] S.P. Kim and W.Y.Su, "Subpixel accuracy image registration by spectrum cancellation," in Proceedings of IEEE International Conference Acoustics, Speech, Signal Processing (ICASSP'93), vol.5, pp.153-156, Minneapolis, Minn, USA, April1993. 